\title{
Recent applications of the Wittig reaction in alkaloid synthesis
}

Majid M. Heravi, Vahideh Zadsirjan, Hoda Hamidi, Mansoureh Daraie, Tayebeh Momeni

Department of Chemistry, School of Science, Alzahra University, Tehran, Iran

\begin{abstract}
The Wittig reaction is the chemical reaction of an aldehyde or ketone with a triphenyl phosphonium ylide (the Wittig reagent) to afford an alkene and triphenylphosphine oxide. Noteworthy, this reaction results in the synthesis of alkenes in a selective and predictable fashion. Thus, it became as one of the keystone of synthetic organic chemistry, especially in the total synthesis of natural products, where the selectivity of a reaction is paramount of importance. A literature survey disclosed the existence of vast numbers of related reports and comprehensive reviews on the applications of this important name reaction in the total synthesis of natural products. However, the aim of this chapter is to underscore, the applications of the Wittig reaction in the total synthesis of one the most important and prevalent classes of natural products, the alkaloids, especially those showing important and diverse biological activities.
\end{abstract}

Keywords: Alkaloids, Wittig reaction, Wittig olefination,Ylides, Total synthesis, Natural products, Olefins 